\title{
Quantum dynamical properties of a two-photon non-linear Jaynes-Cummings model
}

\author{
Guido Berlin and J. Aliaga \\ Departamento de Física, Facultad de Ciencias Exactas y Naturales, \\ Universidad de Buenos Aires, (1428) Buenos Aires, Argentina
}

(October 24, 2018)

\begin{abstract}
In this paper we study a two-photon time-dependent Jaynes-Cummings model interacting with a Kerr-like medium. We assumed that the electromagnetic field is in different states such as coherent, squeezed vacuum and pair coherent, and that the atom is initially in the excited state. We studied the temporal evolution of the population of the excited level, and the second order coherence function. The results obtained show that this system has some similarities with the two-mode Stark system. We analize two photon entanglement for different initial conditions.
\end{abstract}

PACS numbers: 42.50.-p, 42.50.Dv.

Corresponding author: Jorge Aliaga. e-mail: jaliaga@df.uba.ar

Departamento de Física, Facultad de Ciencias Exactas y Naturales, Universidad de Buenos Aires, Pabellón I, (1428) Buenos Aires, Argentina

FAX: $+54-11-4576-3357$

\section{INTRODUCTION}

One of the most fundamental models in quantum optics is the interaction of a single twolevel atom with a single quantized mode of radiation, described by the Jaynes-Cummings 
Hamiltonian [1,2]. Despite being simple enough to be analytically soluble in the rotatingwave approximation, this model is able to describe the quantum-mechanical aspects of the interaction between light and matter. It has led to nontrivial predictions, such as the existence of "collapses" and "revivals" in the atomic excitation [3], and has also allowed a deeper understanding of the dynamical entangling and disentangling of the atom-field

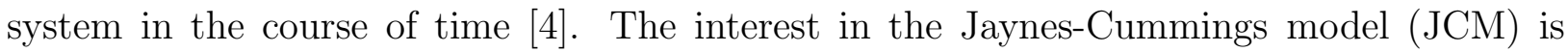
mainly due to the fact that some of its predictions are nowadays accessible to experimental verification [5]. A JCM interaction can be experimentally realized in cavity-QED setups and, as an effective interaction, in laser-cooled trapped ions.

During the last decade many theoretical and experimental efforts have been done in order to study two-photon processes involving atoms inside a cavity, stimulated by the experimental realization of a two-photon cascade micromaser [6] Two-photon processes are also an efficient way of generating squeezed states of the electromagnetic field. It has also been established that two-photon degenerate atoms inside a Kerr-like medium can generate squeezing amplification [7]. The recent discovery of new materials with very high Kerr coupling [8] opened the possibility for the implementation of new experiments that generates entangled states [9] that can be used for a perfect Bell-state discrimination.

A system composed by a three level atom in $\Xi, \Lambda$, and $V$ configurations interacting with two modes of the electromagnetic field was proposed and studied more than ten years ago by Yoo and Eberly [10]. Following these guidelines, Gou [11] investigated the $\Xi$ configuration when the intermediate level can be adiabatically eliminated [12]. This approximation turns the original bilinear photon-level interaction into a three-linear one (usually called "nonlinear non-degenerate two-photon" interaction). This model has been broadly used in order to study the time evolution of the atomic and photon operators, the second order coherence function, the one and two-mode squeezing, the atomic-dipole squeezing and the emission spectra [11, 13] [18]. Usually, the two-level system has been considered initially in the excited state and the two-photons have been chosen initially in two independent coherent states [11, 15, 16, 18], two mode squeezed states [11], pair-coherent states [13], correlated SU $(1,1)$ 
coherent states [14, etc. The $\Lambda$ configuration, also called the Raman coupled model, when the intermediate level can be adiabatically eliminated was studied by Abdalla, Ahmed and Obada [19], and independently by Gerry and Eberly [20]. There have been investigations of the atomic inversion, the appearance of antibunched light, the violations of the CauchySchwartz inequality, population trapping, and squeezing [19 23]. Some similarities in the Rabi frequencies of both configurations for some special conditions of the parameters were reported [14], and the connection between these configurations was deeply studied in Ref. [24]. It was reported that for the $\Xi$ [25,26] and $\Lambda$ 27,28 models, when doing the adiabatic approximation, the appearance of Stark shifts must be taken into account. In Ref. [24] the Stark shifts were neglected in order to separate their contribution to the nontrivial dynamics studied. The intensity-dependent Stark shifts caused by off-resonant levels [29] were modeled by Moya-Cessa, Bužek and Knight [30] using a JCM with an intensity-dependent shift of the two-level transition. Recently, some similarities and differences between the models used in order to describe a cavity filled with a Kerr-like medium, modeled by an anharmonic oscillator, (first analyzed in detail by Jex and Bužek [31] and discussed in many articles thereafter [32, 33, 29, 34]) and Stark effects have been studied [35, 36].

In this paper we study a two-photon time-dependent Jaynes-Cummings model interacting with a nonlinearity that can be, for instance, a Kerr-like medium. The problem is solved using a technique based on obtaining those observables dynamically related [24], and then solving their temporal evolution. We assumed that the electromagnetic field is in different states such as coherent, squeezed vacuum and pair coherent, and that the atom is initially in the excited state. We studied the temporal evolution of the population of the excited level, and the second order coherence function. The results obtained show that this system has some similarities with the two-mode Stark system. 


\section{THE SYSTEM}

We study an effective two-level atom [24], whose levels $\mid g>$ and $\mid e>$, with energies $E_{1}$ and $E_{2}$, interact with two modes of electromagnetic radiation of frequencies $\omega_{1}$ and $\omega_{2}$ inside a non-linear Kerr-like medium. In two-photon processes there are more than two levels involved, but it is possible to neglect them if we assume that $\omega_{1}+\omega_{2} \approx E_{1}-E_{2}(\hbar=1)$ and we consider that the transition frequencies between $|e>| g>$, and the intermediate levels are different from the frequencies of the field. So, the JCM in the rotating wave approximation, ( $\Xi$ configuration), reads [24]:

$$
\hat{H}=\sum_{i=1}^{2} E_{i} \hat{b}_{i}^{\dagger} \hat{b}_{i}+\sum_{i=1}^{2} \omega_{i} \hat{a}_{i}^{\dagger} \hat{a}_{i}+T(t)\left(\gamma \hat{a}_{1} \hat{a}_{2} \hat{b}_{1} \hat{b}_{2}^{\dagger}+\gamma^{*} \hat{b}_{2} \hat{b}_{1}^{\dagger} \hat{a}_{2}^{\dagger} \hat{a}_{1}^{\dagger}\right)
$$

where $a_{i}, a_{i}^{\dagger}, b_{i}^{\dagger}$ and $b_{i}, i=1,2$, are creation and annihilation bosonic and fermionic operators, respectively, and $\gamma$ is the coupling constant between the atomic levels and the fields.

The Kerr medium can be modeled by an anharmonic oscillator coupled to the field 31 33, 29, 34, 37. Using the adiabatic approximation $\left(\omega_{\text {medium }} \ll \omega_{\text {field }}\right)$ the non-linear medium can be represented by a non-linear power of the field. Thus, the total Hamiltonian reads:

$$
\begin{aligned}
\hat{H}= & \sum_{i=1}^{2} E_{i} \hat{b}_{i}^{\dagger} \hat{b}_{i}+\sum_{i=1}^{2} \omega_{i} \hat{a}_{i}^{\dagger} \hat{a}_{i}+T(t)\left(\gamma \hat{a}_{1} \hat{a}_{2} \hat{b}_{1} \hat{b}_{2}^{\dagger}+\gamma^{*} \hat{b}_{2} \hat{b}_{1}^{\dagger} \hat{a}_{1}^{\dagger} \hat{a}_{2}^{\dagger}\right) \\
& +\chi_{1}\left(\hat{a}_{1}^{\dagger}\right)^{2}\left(\hat{a}_{1}\right)^{2}+\chi_{2}\left(\hat{a}_{2}^{\dagger}\right)^{2}\left(\hat{a}_{2}\right)^{2}+2 \sqrt{\chi_{1} \chi_{2}} \hat{a}_{2}^{\dagger} \hat{a}_{1}^{\dagger} \hat{a}_{1} \hat{a}_{2}
\end{aligned}
$$

It is important to notice that three non-linear terms appear due to the presence of the nonlinear Kerr media. Two of them are similar to the ones appearing in the case of one mode while the third one is a bilinear connection between the modes. This bilinear interaction is the one proposed in Refs. [8,9] as a way of generating cat's states.

The dynamical evolution of the mean values of the operators can be obtained by using the Ehrenfest equation,

$$
\frac{d\left\langle\hat{O}_{j}\right\rangle_{t}}{d t}=-\sum_{i} g_{i j}\left\langle\hat{O}_{i}\right\rangle
$$


where $g_{i j}$ are the structure constants of a semi-Lie Algebra closed under commutation with the Hamiltonian [24], i.e.

$$
\left[\hat{H}(t), \hat{O}_{i}\right]=i \hbar \sum_{i} g_{j i}(t) \hat{O}_{j}
$$

The operators defined via the previous equation are called relevant operators ( $\mathrm{RO})$ [38. The relevant operators for the two-modes Jaynes-Cummings Hamiltonian read

$$
\begin{aligned}
\hat{N}_{1}^{n, m} & \equiv\left(\hat{a}_{1}^{\dagger}\right)^{n}\left(\hat{a}_{2}^{\dagger}\right)^{m} \hat{b}_{1}^{\dagger} \hat{b}_{1}\left(\hat{a}_{2}\right)^{m}\left(\hat{a}_{1}\right)^{n} \\
\hat{N}_{2}^{n, m} & \equiv\left(\hat{a}_{1}^{\dagger}\right)^{n}\left(\hat{a}_{2}^{\dagger}\right)^{m} \hat{b}_{2}^{\dagger} \hat{b}_{2}\left(\hat{a}_{2}\right)^{m}\left(\hat{a}_{1}\right)^{n} \\
\hat{\Delta}_{1}^{n, m} & \equiv\left(\hat{a}_{1}^{\dagger}\right)^{n}\left(\hat{a}_{2}^{\dagger}\right)^{m} \hat{a}_{1}^{\dagger} \hat{a}_{1}\left(\hat{a}_{2}\right)^{m}\left(\hat{a}_{1}\right)^{n} \\
\hat{\Delta}_{2}^{n, m} & \equiv\left(\hat{a}_{1}^{\dagger}\right)^{n}\left(\hat{a}_{2}^{\dagger}\right)^{m} \hat{a}_{2}^{\dagger} \hat{a}_{2}\left(\hat{a}_{2}\right)^{m}\left(\hat{a}_{1}\right)^{n} \\
\hat{I}^{n, m} & \equiv\left(\hat{a}_{1}^{\dagger}\right)^{n}\left(\hat{a}_{2}^{\dagger}\right)^{m}\left(\gamma \hat{a}_{1} \hat{a}_{2} \hat{b}_{1} \hat{b}_{2}^{\dagger}+\gamma^{*} \hat{a}_{1}^{\dagger} \hat{a}_{2}^{\dagger} \hat{b}_{1}^{\dagger} \hat{b}_{2}\right)\left(\hat{a}_{2}\right)^{m}\left(\hat{a}_{1}\right)^{n} \\
\hat{F}^{n, m} & \equiv\left(\hat{a}_{1}^{\dagger}\right)^{n}\left(\hat{a}_{2}^{\dagger}\right)^{m} i\left(\gamma \hat{a}_{1} \hat{a}_{2} \hat{b}_{1} \hat{b}_{2}^{\dagger}-\gamma^{*} \hat{a}_{1}^{\dagger} \hat{a}_{2}^{\dagger} \hat{b}_{1}^{\dagger} \hat{b}_{2}\right)\left(\hat{a}_{2}\right)^{m}\left(\hat{a}_{1}\right)^{n}, \\
\hat{N}_{2,1}^{n, m} & \equiv\left(\hat{a}_{1}^{\dagger}\right)^{n}\left(\hat{a}_{2}^{\dagger}\right)^{m} \hat{b}_{2}^{\dagger} \hat{b}_{2} \hat{b}_{1}^{\dagger} \hat{b}_{1}\left(\hat{a}_{2}\right)^{m}\left(\hat{a}_{1}\right)^{n} .
\end{aligned}
$$

Notice that $\hat{I}^{n, m}$ and $\hat{F}^{n, m}$ are the operators that have the information about the correlation between the modes and the atom levels (i.e. the entanglement).

Using the Ehrenfest theorem, the evolution equations of the relevant operators are

$$
\begin{aligned}
& \frac{d\left\langle\hat{N}_{1}^{n, m}\right\rangle}{d t}=T(t) \hat{F}^{n, m}+n T(t) \hat{F}^{n-1, m}+m T(t) \hat{F}^{n, m-1} \\
& +n m T(t) \hat{F}^{n-1, m-1}, \\
& \frac{d\left\langle\hat{N}_{2}^{n, m}\right\rangle}{d t}=-T(t) \hat{F}^{n, m} \text {, } \\
& \frac{d\left\langle\hat{\Delta}_{1}^{n, m}\right\rangle}{d t}=(n+1) T(t) \hat{F}^{n, m}+m T(t) \hat{F}^{n, m-1} \\
& +m T(t) \hat{F}^{n+1, m-1}+n m T(t) \hat{F}^{n, m-1}, \\
& \frac{d\left\langle\hat{\Delta}_{2}^{n, m}\right\rangle}{d t}=(m+1) T(t) \hat{F}^{n, m}+n T(t) \hat{F}^{n-1, m} \\
& +n T(t) \hat{F}^{n-1, m+1}+n m T(t) \hat{F}^{n-1, m}, \\
& \frac{d\left\langle\hat{N}_{2,1}^{n, m}\right\rangle}{d t}=0
\end{aligned}
$$




$$
\begin{aligned}
\frac{d\left\langle\hat{I}^{n, m}\right\rangle}{d t}= & {\left[\alpha-2\left(n \chi_{1}+m \chi_{2}+(m+n+1) \sqrt{\chi_{1} \chi_{2}}\right)\right] \hat{F}^{n, m} } \\
& -2\left(\chi_{1}+\sqrt{\chi_{1} \chi_{2}}\right) \hat{F}^{n+1, m}-2\left(\chi_{2}+\sqrt{\chi_{1} \chi_{2}}\right) \hat{F}^{n, m+1} \\
\frac{d\left\langle\hat{F}^{n, m}\right\rangle}{d t}= & -\left[\alpha-2\left(n \chi_{1}+m \chi_{2}+(m+n+1) \sqrt{\chi_{1} \chi_{2}}\right)\right] \hat{I}^{n, m} \\
& +2\left(\chi_{1}+\sqrt{\chi_{1} \chi_{2}}\right) \hat{I}^{n+1, m}+2\left(\chi_{2}+\sqrt{\chi_{1} \chi_{2}}\right) \hat{I}^{n, m+1} \\
& +2|\gamma|^{2} T(t)\left[(n+1)(m+1) \hat{N}_{2}^{n, m}-(n+1)(m+1) \hat{N}_{2,1}^{n, m}\right. \\
& +(n+1) \hat{N}_{2}^{n, m+1}-(n+1) \hat{N}_{2,1}^{n, m+1}+(m+1) \hat{N}_{2}^{n+1, m} \\
& \left.-(m+1) \hat{N}_{2,1}^{n+1, m}-\hat{N}_{1}^{n+1, m+1}+\hat{N}_{2}^{n+1, m+1}\right] .
\end{aligned}
$$

where $\alpha=E_{2}-E_{1}-\omega_{1}-\omega_{2}$.

So, using Eqs. (6) it is possible to evaluate the temporal evolution of the RO. These operators close a Lie Algebra. Thus, any extension of the two-photon JCM built up adding terms proportional to RO will have similar evolution equations. This fact will be used in the following section in order to study the two-photon JCM with Stark shifts.

Equations (6) can be numerically solved for any temporal dependence of the atomic-field interaction, $T(t)$. For the time-independent case, Eqs. (河) can be analytically solved using the series expansion in terms of commutators with the Hamiltonian [24,34,38

$$
\langle\hat{O}\rangle_{t}=\langle\hat{O}\rangle_{0}+\sum_{n \geq 0} \frac{1}{n !}\left(\frac{t}{i \hbar}\right)^{n}\langle[\ldots[\hat{O}, \hat{H}], \ldots \ldots, \hat{H}]\rangle_{0} .
$$

From Eqs. (6) we can notice that the evolution equations for all the operators different from $\hat{F}^{n, m}$ depend on $\hat{F}^{n, m}$. So, the double commutator $\left[\hat{H},\left[\hat{H}, \hat{F}^{n, m}\right]\right]$ plays a central role. This double commutator reads

$$
\begin{aligned}
{\left[\hat{H},\left[\hat{H}, \hat{F}^{n, m}\right]\right]=} & \beta_{n, m}^{2} \hat{F}^{n, m}+\left(\beta_{n+1, m}^{2}-\beta_{n, m}^{2}\right) \hat{F}^{n+1, m}+ \\
& \left(\beta_{n, m+1}^{2}-\beta_{n, m}^{2}\right) \hat{F}^{n, m+1}+\left[8 \epsilon_{1} \epsilon_{2}+4|\gamma|^{2}\right] \hat{F}^{n+1, m+1}+ \\
& 4 \epsilon_{1}^{2} \hat{F}^{n+2, m}+4 \epsilon_{2}^{2} \hat{F}^{n, m+2}
\end{aligned}
$$

where the generalized Rabi frequency $\beta_{n, m}^{2}=\left[\alpha-2\left(n \chi_{1}+m \chi_{2}+(n+m+1) \sqrt{\chi_{1} \chi_{2}}\right)\right]^{2}+$ $4|\gamma|^{2}(n+1)(m+1)$ and $\epsilon_{i}=\chi_{i}+\sqrt{\chi_{1} \chi_{2}}$. It is important to notice that the term propor- 
tional to $\sqrt{\chi_{1} \chi_{2}}$ appearing in $\beta_{n, m}^{2}$ and $\epsilon_{i}$ is due to the bilinear connection between modes. Equation (8) generates paths, or different ways of dynamically connecting the RO, representing quantum correlations [24, 34, 36, 38]. From Eq. (8) it can be seen that those terms proportional to $\epsilon_{i}$ are due to the presence of the Kerr medium. Notice that taking into account the bilinear connection between modes doubles the value of $\epsilon_{i}$ in the $\chi_{1}=\chi_{2}$ case usually studied.

Making use of Eq. (8) we can obtain the temporal evolution of the population of the excited level $\left(\left\langle\hat{N}_{2}^{0,0}\right\rangle_{t}\right)$ and the second order intermodes coherence function (defined as $g_{12}^{2}(t)=\frac{\left\langle\hat{a}_{1}^{\dagger} \hat{a}_{1} \hat{a}_{2}^{\dagger} \hat{a}_{2}\right\rangle}{\left\langle\hat{a}_{1}^{\dagger} \hat{a}_{1}\right\rangle\left\langle\hat{a}_{2}^{\dagger} \hat{a}_{2}\right\rangle}-1$ ), for different states of the field, (See appendix).

This is depicted in Figs. 11-3. In all cases, we assume the atom initially in the excited state $\left(\left\langle\hat{N}_{1}^{0,0}\right\rangle(0)=0,\left\langle\hat{N}_{2}^{0,0}\right\rangle(0)=1\right)$. The phenomenological third-oder nonlinear susceptibility for the Kerr media $\chi=\chi_{1}=\chi_{2}$ takes the values $0,0.5$ and 1 , which, in principle, could be obtained in the ultra-high susceptibility Kerr cells recently studied [8]. We also assume that there are initially 10 photons in each mode $\left(\left|\alpha_{i}\right|^{2}=10\right)$. We analyze the influence of the non-linear medium in the temporal evolution of the excited level and we find inhibited decay as it can be seen from Figs. 1-b,2-b,3-b. In the Pair Coherent state case (Fig. 3-b), we find that the revivals are of such a kind that the atom recovers its initial population in each revival, and this effect is increased as we make more important the coupling with the non-linear medium. Another interesting feature is that in the squeezed vacuum state (Fig. 2-b) we see that the revivals are regular and sharp, and become more periodic as we increase the strength of the coupling with the Kerr medium. We can see from Figs. 1 F a and 3-a, that the two-photon JCM presents antibunching, and that this effect is attenuated by a stronger interaction with the non-linear medium. In the particular case of the squeezed vacuum, we notice that the field recovers its initial intermodes coherence when the excited level population reaches a maximum.

Finally, we want to mention that $\Lambda$ configuration can be studied by using the canonical transformation $\hat{\tilde{a}}_{1}=\hat{a}_{1}^{\dagger}$ and redefining the RO following Ref. [24]. 


\section{STARK EFFECT}

The Stark effect is related to changes in the atomic energy levels due to virtual transitions from levels out of resonance. The effective Hamiltonian taking into account the Stark effect reads

$$
\begin{aligned}
\hat{H}= & \sum_{i=1}^{2} E_{i} \hat{b}_{i}^{\dagger} \hat{b}_{i}+\sum_{i=1}^{2} \omega_{s_{i}} \hat{a}_{i}^{\dagger} \hat{a}_{i}+T(t)\left(\gamma \hat{a}_{1} \hat{a}_{2} \hat{b}_{1} \hat{b}_{2}^{\dagger}+\gamma^{*} \hat{b}_{2} \hat{b}_{1}^{\dagger} \hat{a}_{2}^{\dagger} \hat{a}_{1}^{\dagger}\right) \\
& +\hat{a}_{1}^{\dagger} \hat{a}_{1}\left(\eta_{1} \hat{b}_{1}^{\dagger} \hat{b}_{1}+\eta_{2} \hat{b}_{2}^{\dagger} \hat{b}_{2}\right)+\hat{a}_{2}^{\dagger} \hat{a}_{2}\left(\eta_{1} \hat{b}_{1}^{\dagger} \hat{b}_{1}+\eta_{2} \hat{b}_{2}^{\dagger} \hat{b}_{2}\right)
\end{aligned}
$$

Both the Kerr Hamiltonian and the Stark Hamiltonian can be written in terms of RO (for the one-mode case see Ref. [36]. Using the same technique is is easy to see that if

$$
\begin{aligned}
\omega_{1}+\sqrt{\chi_{1} \chi_{2}} & =\omega_{s_{1}}+\eta_{1} \\
\omega_{2}+\sqrt{\chi_{1} \chi_{2}} & =\omega_{s_{2}}+\eta_{2} \\
\eta_{1}-\eta_{2} & =2 \epsilon_{1}=2 \epsilon_{2},
\end{aligned}
$$

the evolution equations for the relevant operators will be the same. In this case, for the RO here studied, both systems will have the same temporal evolution [36]. This will not be the case if we study the temporal evolution of other R.O. like the electromagnetic field $\left(\hat{a}+\hat{a}^{\dagger}\right)$.

\section{CONCLUSION}

In the present paper we analyzed the non-degenerate two-photon JCM in the presence of a Kerr media. We have identified those relevant operators that are interrelated with the dynamics of the system. We find that the dynamical evolution of the relevant operators for this problem and for the model that takes into account Stark shifts are the same for these RO and for some values of the constants. We analyze the influence of the non-linear medium in the temporal evolution of the excited level and we find inhibited decay. In the Pair Coherent state case, we observe that the revivals are of such a kind that the modes of the field are disentangled, and this effect is increased as we make more important the 
coupling with the non-linear medium. We also notice that in the squeezed vacuum state the revivals are regular and sharp, and that the modes of the field are entangled. We also see that this system presents antibunching, and that this effect is attenuated by a stronger interaction with the non-linear medium. Thus, this system can be used in order to obtain very different two photon entanglement situations, depending on the initial state of the field that is considered. This fact can be very useful in experimental realizations.

\section{ACKNOWLEDGMENTS}

The authors acknowledge support from Consejo Nacional de Investigaciones Científicas y Técnicas (Conicet), Argentina.

\section{APPENDIX: EXACT EXPRESSIONS FOR THE TIME EVOLUTION OF THE RELEVANT OPERATORS}

In the time independent case, making use of equations (8,7) we can obtain an exact expression for all the Relevant Operators. For instance $\left\langle\hat{N}_{1}^{0,0}\right\rangle(t)$ reads:

$$
\begin{aligned}
\left\langle\hat{N}_{1}^{0,0}\right\rangle(t)=\left\langle\hat{N}_{1}^{0,0}\right\rangle(0) & -\sum_{j, k=0}^{\infty} \frac{S_{j, k}}{\beta_{j, k}} \sum_{n=j}^{\infty} \sum_{m=k}^{\infty} a_{j, k}^{n, m}\left\langle\hat{F}^{n, m}\right\rangle_{0} \\
& -\sum_{j, k=0}^{\infty} \frac{C_{j, k}}{\beta_{j, k}^{2}} \sum_{n=j}^{\infty} \sum_{m=k}^{\infty} a_{j, k}^{n, m}\left[2 \epsilon_{1}+2 \epsilon_{2}-\left[\alpha-2\left(n \epsilon_{1}+m \epsilon_{2}\right.\right.\right. \\
& \left.\left.\left.+\sqrt{\chi_{1} \chi_{2}}\right)\right]\right]\left\langle\hat{I}^{n, m}\right\rangle_{0}+2|\gamma|^{2}\left[\left\langle\hat{N}_{2}^{n+1, m+1}\right\rangle-\left\langle\hat{N}_{1}^{n+1, m+1}\right\rangle\right] \\
& +2|\gamma|^{2}(n+1)(m+1)\left[\left\langle\hat{N}_{2}^{n, m}\right\rangle-\left\langle\hat{N}_{2,1}^{n, m}\right\rangle\right] \\
& +2|\gamma|^{2}(n+1)\left[\left\langle\hat{N}_{2}^{n, m+1}\right\rangle-\left\langle\hat{N}_{2,1}^{n, m+1}\right\rangle\right]
\end{aligned}
$$

where $S_{j, k}=\sin \left(\beta_{j, k} t\right), C_{j, k}=\cos \left(\beta_{j, k} t\right)-1$, and $a_{j, k}^{n, m}=(-1)^{n+m+j+k+1} /[(n-j) ! j !(m-k) ! k !]$. We can evaluate this expression for different initial conditions for the population of the atomic levels, and the state of the field.

If we assume that the field is initially in a coherent state, 


$$
\left|\alpha_{1} \alpha_{2}>=\sum_{i=0}^{\infty} P_{n_{1}} P_{n_{2}}\right| n_{1} n_{2}>
$$

where

$$
P_{n_{i}}=\frac{e^{|\alpha|^{2}}|\alpha|^{2^{n_{i}}}}{n_{i} !}
$$

then the expression (A1) takes the form:

$$
\left\langle\hat{N}_{1}^{0,0}\right\rangle_{t}=1+2|\gamma|^{2} \sum_{j, k=0}^{\infty} \frac{1}{j ! k !} \frac{C_{j, k}}{\beta_{j, k}^{2}}\left\langle\hat{\Delta}_{1}^{0,0}\right\rangle_{0}^{j}\left\langle\hat{\Delta}_{2}^{0,0}\right\rangle_{0}^{k} e^{-\left\langle\hat{\Delta}_{1}^{0,0}\right\rangle_{0}} e^{-\left\langle\hat{\Delta}_{2}^{0,0}\right\rangle_{0}}
$$

where $\Delta_{i}^{0,0}(i=1,2)$ is the number of photons present in each mode of the field.

The same treatment can be applied to the case of having the field in the Squeezed Vacuum state [39], which can be written in the following way,

$$
\left|r, \phi>=(\cosh (r))^{-1} \sum_{n}\left(e^{i \phi} \tanh (r)\right)^{n}\right| n, n>
$$

In this case the expression (A1) reads,

$$
\left\langle\hat{N}_{1}^{0,0}\right\rangle_{t}=1+\frac{2|\gamma|^{2}}{\cosh ^{2}(r)} \sum_{k=0}^{\infty}(k+1)^{2} \tanh ^{2}(r) \frac{C_{k, k}}{\beta_{k, k}^{2}}
$$

Finally, we assume the field is in the Pair Coherent state 40],

$$
\left|\xi, q>=N_{q} \sum_{n=0}^{\infty} \frac{\xi^{n}}{[n !(n+q) !)]^{\frac{1}{2}}}\right| n+q, n>,
$$

where $N_{q}$ is a normalization constant. In this case we have:

$$
\left\langle\hat{N}_{1}^{0,0}\right\rangle_{t}=1+2|\gamma|^{2} N_{q} \sum_{k=0}^{\infty}(k+1)(k+q+1) \frac{|\xi|^{2 k}}{k !(k+q) !} C_{k, k}
$$




\section{REFERENCES}

[1] E.T. Jaynes and F.W. Cummings, Proc. IEEE 51, 89 (1963).

[2] B.W. Shore and P.L. Knight, J. Mod. Opt.40, 1195 (1993).

[3] J.H. Eberly, N.B. Narozhny, and J.J.Sanchez-Mondragon, Phys. Rev. Lett. 44, 1323 (1980); N.B. Narozhny, J.J. Sanchez-Mondragon, and J.H. Eberly, Phys. Rev. A 23, 236 (1981); H.I. Yoo, J.J. Sanchez-Mondragon and J.H. Eberly, J. Phys A 14 ,1383 (1981).

[4] S.J.D. Phoenix and P.L. Knight, Ann. Phys. (N.Y.), 186, 381 (1988); Phys. Rev. A 44, 6023 (1991); A. Ekert and P.L. Knight, Am. J. Phys. 63, 415 (1995).

[5] D. Meschede, H. Walther, and G. Muller, Phys. Rev. Lett. 54 (1985) 551; G. Rempe, H. Walther, and N. Klein, ibid. 58 (1987) 353; G. Rempe, F. Schmidt-Kaler, and H. Walther, ibid. 64 (1990) 2783; G. Rempe, R. J. Thompson, R. J. Brecha, W. D. Lee, and H. J. Kimble, ibid. 67 (1991) 1727; F. Bernardot, P. Nussenzveig, M. Brune, J. M. Raimond, and S. Haroche, Europhys. Lett. 17 (1992) 33; R. J. Thompson, G. Rempe, and H. J. Kimble, Phys. Rev. Lett. 68 (1992) 1132; C. Weisbuch, M. Nishioka, A. Isikawa, and Y. Arakawa, ibid. 69 (1992) 3314; L. Davidovich, A. Maali, M. Brune, J. M. Raimond, and S. Haroche, ibid. 71 (1993) 2360; O. Benson, G. Raitel, and H. Walther, ibid. 72 (1994) 3506; G. Raithel, O. Benson, and H. Walther, ibid. 75 (1995) 3446; M. Brune, F. Schmidt-Kaler, A. Maali, J. Dreyer, E. Hagley, J. M. Raimond, and S. Haroche, ibid. 76 (1996) 1800.

[6] M. Brune, J. M. Raimond, P. Goy, L. Davidovich, and S. Haroche, Phys. Rev. Lett. 59 (1987) 1899.

[7] Si-de Du, Shang-qing Gong, Zhi-zhan Xu, Lu-wei Zhou, Chang-de Gong, Opt. Comm. L38 (1997) 193; Li Gaoxiang, Peng Jinsheng, Zhou Peng, Chin. Phys. Lett. 12 (1995) 79. 
[8] L. Vestergaard Hau et al., Nature 397 (1999) 594; S.E. Harris and L.V. Hau, Phys. Rev. Lett. 82 (1999) 4611; U. Rathe et al, Phys. Rev. A 47 (1993) 4994; M.M. Kash et al., Phys. Rev. Lett. 82 (1999) 5229.

[9] David Vitali, Mauro Fortunato, and Paolo Tombesi, Phys. Rev. Lett. 85 (2000) 445;

M. Genovese and C. Novero, Phys.Lett. A 271 (2000) 48.

[10] H.-I. Yoo and J.H. Eberly, Phys. Rep. 118 (1985) 239.

[11] S.-C. Gou, Phys. Rev. A 40 (1989) 5116; ibid 41 (1991) 4097.

[12] P. Alsing and M. S. Zubairy, J. Opt. Soc. Am. B4 (1987) 177.

[13] A. Joshi and R. R. Puri, Phys. Rev. A 42 (1990) 4336.

[14] C. C Gerry and R. F. Welch, J. Opt. Soc. Am. B9 (1992) 290.

[15] A. M. Abdel-Hafez, Phys. Rev. A 45 (1992) 6610.

[16] M. M Ashraf and M. S. K. Razmi, Phys. Rev. A 45 (1992) 8121; J. Mod. Opt. 39 (1992) 2245.

[17] M. M Ashraf, Phys. Rev. A 50 (1994) 5116.

[18] H. Iwasawa, K. Matsuo, Opt. Comm. 177 (1995) 551.

[19] M. S. Abdalla, M. M. A. Ahmed, and A.-S. F Obada, Physica A162 (1990) 215.

[20] C. Gerry and J. Eberly, Phys. Rev. A 42 (1990) 6805.

[21] D. A. Cardimona, V. Kovanis, M. P. Sharma, and A. Gavrielides, Phys. Rev. A 43 (1991) 3710 .

[22] C. Gerry and H. Huang, Phys. Rev. A 45 (1992) 8037.

[23] B. Deb, G. Gangopadhyay, and D. S. Ray, Phys. Rev. A 48 (1993) 1400; ibid. 51 (1995) 2651. 
[24] J. Aliaga and J.L. Gruver, Phys. Lett. A 221, (1996) 19.

[25] S.-C. Gou, Phys. Lett A 147 (1990) 218; Phys. Rev. A 48 (1993) 3233.

[26] R. D'souza and A. Jayarao, Mod. Phys. Lett. B 9 (1995) 665.

[27] T. Nasreen, J. Opt. Soc. Am. B 11 (1994) 386.

[28] G.-X. Li and J.-S. Peng, Phys. Rev. A 52 (1995) 465.

[29] A. Joshi and S. V. Lawande, Phys. Rev. A 46, 5906 (1992).

[30] H. Moya-Cessa, V Bužek and P. L. Knight, Optics Commun. 85, 267 (1991).

[31] V. Bužek and I. Jex, Opt. Commun. 78, 425 (1990).

[32] M. J. Werner and H. Risken, Quantum Opt. 3, 185 (1991).

[33] Ho Trung Dung and A. S. Shumovsky, Phys. Lett. A 160, 437 (1991).

[34] J. L. Gruver, J. Aliaga, Hilda A. Cerdeira, and A. N. Proto, Phys. Lett. A 190 , (1994) 363.

[35] H. Moya-Cessa, S. Chávez-Cerda, J. J. Sánchez-Mondragón, Phys. Lett. A 205, 51 (1995).

[36] J.L. Gruver and J. Aliaga Phys. Rev. A 56, (1997) 2473

[37] N. Imoto, H.A. Haus, Y. Yamamoto, Phys. Rev. A 32, (1985) 2287.

[38] J.L. Gruver, J Aliaga, Hilda A. Cerdeira y A. N. Proto, Phys Rev A 50, (1994) 5274

[39] D. F. Walls, G. J. Milburn, Quantum Optics, (Springer-Verlag, Berlin, 1994).

[40] G.S. Agarwal, J. Opt. Soc. Am. B 5, 1940 (1988) 


\section{FIGURES}

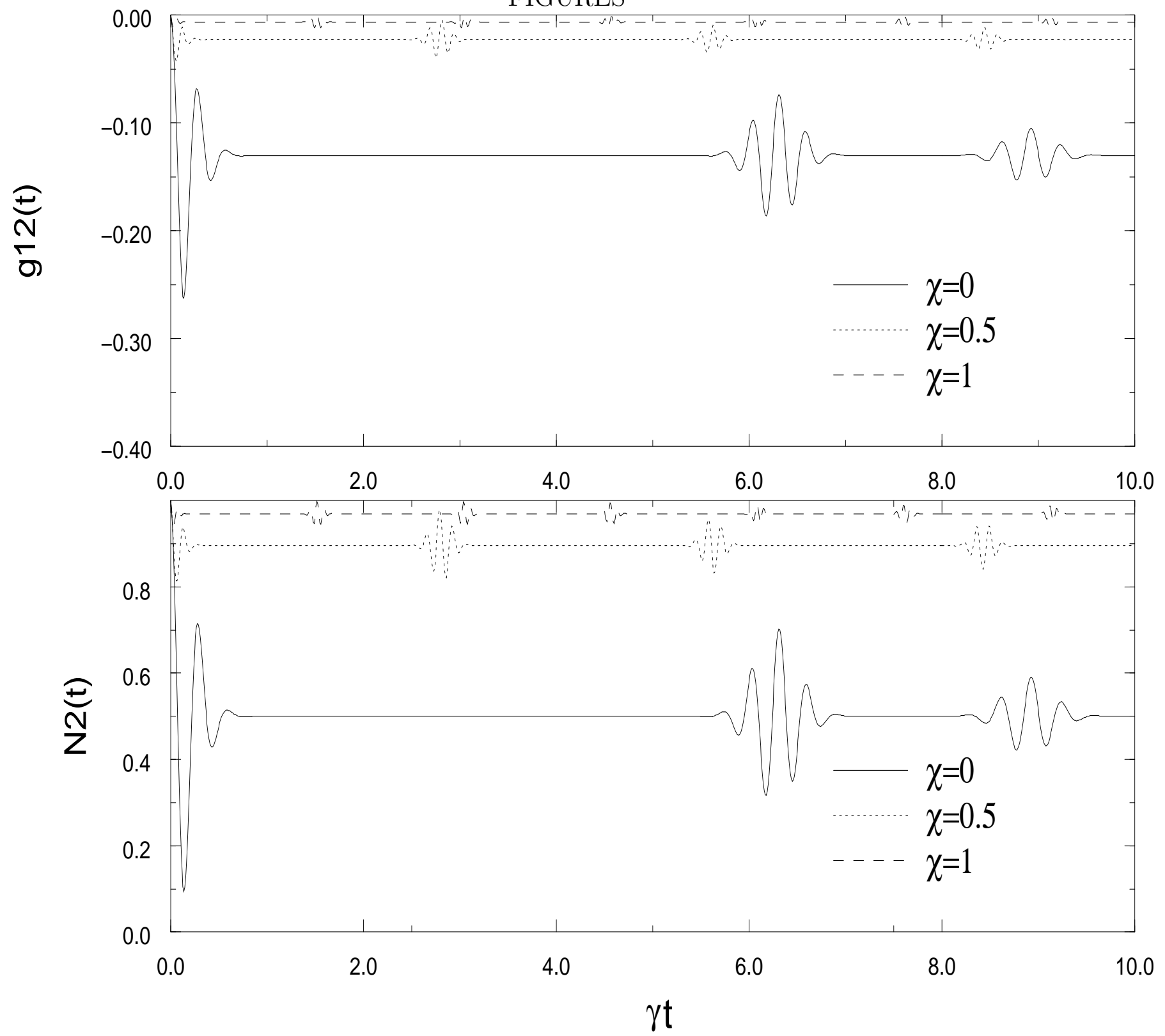

FIG. 1. Figure 1. $\left\langle\hat{N}_{2}^{0,0}\right\rangle(t)$ and $g_{12}^{2}(t)$ for coherent state field, $\left\langle\hat{N}_{2}^{0,0}\right\rangle(0)=1,\left\langle\hat{N}_{1}^{0,0}\right\rangle(0)=0$ and $\left.\left|\alpha_{i}\right|^{2}=10\right)$ 

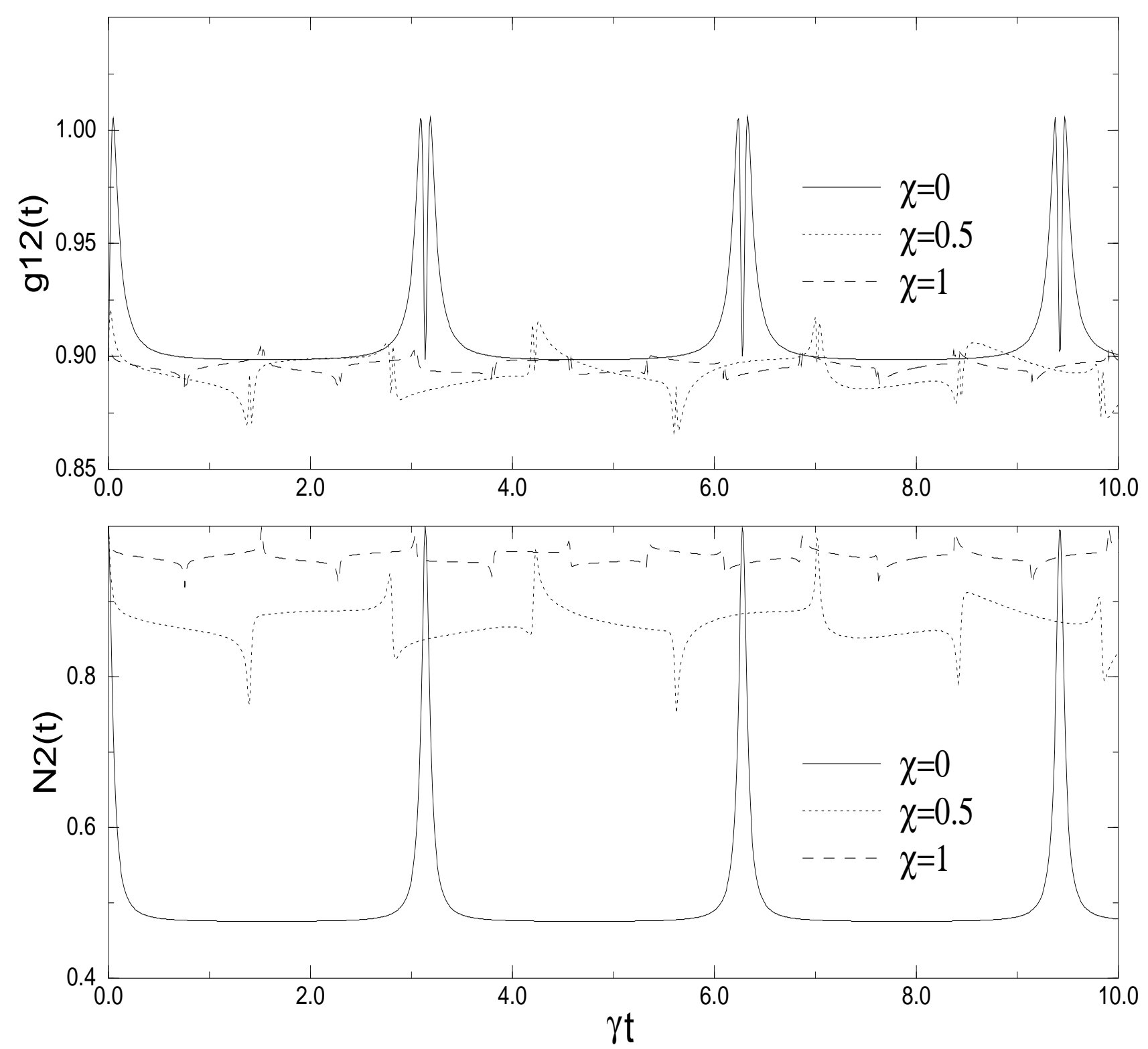

FIG. 2. Figure 2. Same as figure 1 but field in squeezed vacuum. 

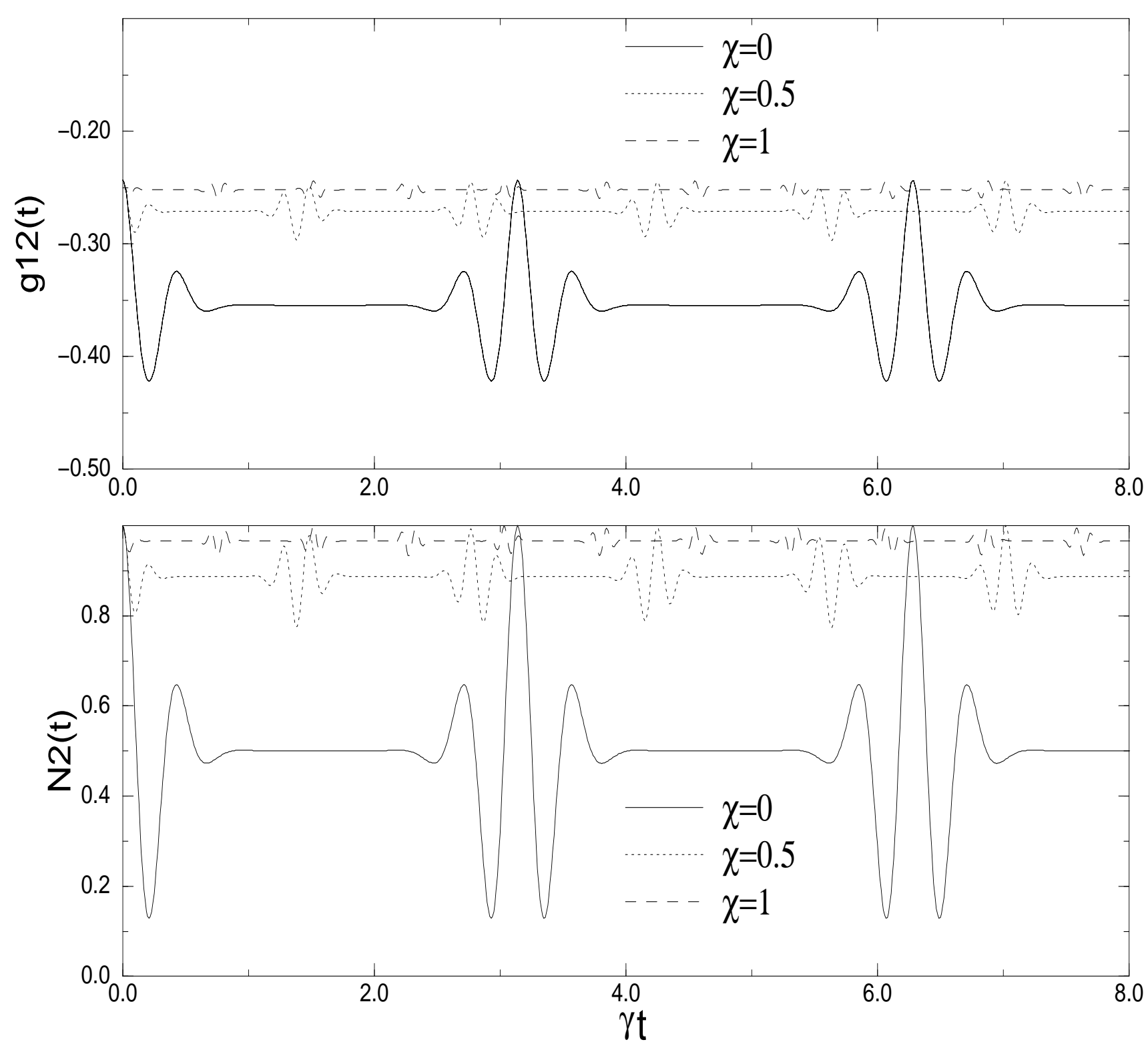

FIG. 3. Figure 3. Same as figure 1 but field in pair coherent field. 